BULL. AUSTRAL. MATH. SOC.

\title{
INVERSE LIMIT THEOREM AND EXPANSION THEOREM FOR COHOMOLOGICAL DIMENSION
}

\author{
Satya Deo and Subhash Muttepawar
}

\begin{abstract}
For a given ground ring $L$, let $\operatorname{dim}_{L}(X)$ denote the sheaf theoretic cohomological dimension of a space $X$. In this paper we prove an inverse limit theorem for this dimension function. Then we apply this theorem to show that for a large class of rings the Freudenthal's expansion theorem, expressing a compact metric space $X$ of $\operatorname{dim}_{L}(X)=n$ as the inverse limit of an system of compact polyhedra $K_{\alpha}$ of $\operatorname{dim}_{L}\left(K_{\alpha}\right) \leq n$, is not valid.
\end{abstract}

\section{Introduction and preliminaries}

Let $X$ be the inverse limit of an inverse system $\left\{X_{\alpha}, \pi_{\alpha, \beta}\right\}_{\alpha, \beta \in \Omega}$ of topological spaces $X_{\alpha}$ and continuous maps $\pi_{\alpha, \beta}: X_{\beta} \rightarrow X_{\alpha}, \alpha<\beta$, and $d$ be a dimension function defined for a class of spaces containing $X_{\alpha}$ and $X$. Then a theorem stating conditions on spaces $X_{\alpha}$ and mappings $\pi_{\alpha, \beta}$ which ensure that

$$
d(X) \leq \sup _{\alpha}\left\{d\left(X_{\alpha}\right)\right\}
$$

is called an inverse limit theorem for dimension function $d$. Recently Katuta [6] obtained many inverse limit theorems for the covering dimension (dim) which generalize the earlier known theorems. Nagami has also obtained an inverse limit theorem for the Katětov dimension [8]; that the

Received 2 April 1983. 
inverse limit theorem is not generally valid for the Katētov dimension has been shown by Charalambous [2]. Let $X$ be any space such that $d(X) \leq n$. Then a theorem stating conditions on $X$ which ensure that $X$ can be expressed as the inverse limit of an inverse system $\left\{x_{\alpha}, \pi_{\alpha, \beta}\right\}$ consisting of 'nice spaces' $X_{\alpha}$ such that $d\left(X_{\alpha}\right) \leq n$, is called an expconsion theorem for the dimension function $d$. The first expansion theorem for the covering dimension was proved by Freudenthal as follows. If $X$ is a compact metric space such that $\operatorname{dim} X=n$, then $X$ can be expressed as the inverse limit of an inverse system $\left\{K_{\alpha}, \pi_{\alpha, \beta}\right\}$, consisting of compact polyhedra $K_{\alpha}$ such that dim $K_{\alpha} \leq n$. That such an expansion theorem is not true for an arbitrary compact Hausdorff space was shown by Mardešić [7]. Now it is natural to ask whether or not, for a given ring $L$, these inverse limit theorems and the expansion theorems are valid for sheaf theoretic cohomological dimension $\left(\mathrm{dim}_{L}\right)$. To be specific, let us ask the following. Suppose $L$ is a ring and $X$ is a compact metric space such that $\operatorname{dim}_{L}(X)=n$. Does there exist an inverse system $\left\{K_{\alpha}, \pi_{\alpha, \beta}\right\}$ consisting of compact polyhedra $K_{\alpha}$ such that $\operatorname{dim}_{L}\left(K_{\alpha}\right) \leq n$ and $X$ is homeomorphic to the inverse limit of $\left\{k_{\alpha}, \pi_{\alpha, \beta}\right\}$ ? If $L$ is a ring such that for all compact Hausdorff spaces $\operatorname{dim}_{L}(X)=\operatorname{dim} X$, then the answer to the above question is evidently trivial. But for a given ring $L$, it may not be the case; in fact for $L=\mathbb{Z}$ that is the famous problem of Aleksandrov which is still open. Hence the answer to the above question has to be attempted directly without making use of similar known results for the covering dimension. The main objective of this paper is to show that for a large number of rings $L$ (for example, $L=\mathbb{Z}_{p}, p$ a prime) the Freudenthal's theorem is not valid for sheaf theoretic cohomological dimension $\operatorname{dim}_{L}$. It would be, of course, desirable to determine those rings $L$ for which Freudenthal's theorem is true. The theorem may be true for the case when $L=\mathbb{Z}$. We have, however, no positive results in this direction.

Let $X$ be any locally paracompact space. Then $X$ admits a paracompactifying family $\varphi$ of supports such that $U\{K \mid K \in \varphi\}=X$. For a given ring $L$, the largest integer $n$ (or $\infty$ ) for which there exists a 
sheaf $A$ of $L$-modules on $X$ such that $H_{\varphi}^{n}(X ; A) \neq 0$, is called the (sheaf theoretic) cohomological dimension of $X$ and is denoted by $\operatorname{dim}_{L}(X)$. For additional details and further results about $\operatorname{dim}_{L}$ we refer to ([1], [3], [4] and [5]). All notations are from [1], and in this paper $L$ stands for a non-trivial principal ideal domain. We will make use of the following Floyd-Grothendieck characterization of $\operatorname{dim}_{L}(X)$ for any locally compact Hausdorff space $X \quad([1], \mathrm{p} .72): \operatorname{dim}_{L}(X) \leq n$ if and only if $H_{c}^{n+1}(U ; L)=0$ for each open subset $U$ of $X$.

\section{An inverse limit theorem}

For any locally compact Hausdorff space $X$, let $\hat{X}$ denote the onepoint compactification of $X$. We start with the following observation: if $\left\{X_{\alpha}, \pi_{\alpha, \beta}\right\}$ is an inverse system of locally compact Hausdorff spaces and perfect bonding maps $\pi_{\alpha, \beta}$ such that $X$ is homeomorphic to the inverse limit of $\left\{x_{\alpha}, \pi_{\alpha, \beta}\right\}$, then one can easily verify that $\hat{X}$ will be homeomorphic to the inverse limit of the inverse system $\left\{\hat{X}_{\alpha}, \hat{\pi}_{\alpha, \beta}\right\}$, that is, the one-point compactification commutes with taking inverse limit of locally compact Hausdorff spaces with perfect bonding maps. We also note that for any ring $L, \operatorname{dim}_{L}(X)=\operatorname{dim}_{L}(\hat{X})$; this follows directly or from the complementary sum theorem proved in [5].

Let us now prove the following inverse limit theorem for the cohomological dimension.

THEOREM 1. Let $\left\{X_{\alpha}, \pi_{\alpha, \beta}\right\}_{\alpha, \beta \in \Omega}$ be an inverse system of locally compact Hausdorff spaces with perfect bonding maps and $X={ }_{+} \lim X_{\alpha}$. Then for any $L$,

$$
\operatorname{dim}_{L}(X) \leq \sup _{\alpha}\left\{\operatorname{dim}_{L}\left(x_{\alpha}\right)\right\}
$$

Proof. By taking one-point compactifications we can assume, by our remark made above, that $X_{\alpha}$ is compact for each $\alpha$. It follows that $X$ is also compact Hausdorff. Suppose $\operatorname{dim}_{L}\left(x_{\alpha}\right) \leq n$ for each $\alpha$. By the Floyd-Grothendieck characterization it suffices to prove that 
$H_{c}^{n+1}(U ; L)=0$ for each open set $U$ of $X$. Let $\pi_{\alpha}: X \rightarrow X_{\alpha}$ be the canonical projections for each $\alpha$. First of all we claim that $H_{c}^{n+1}\left(\pi_{\alpha}^{-1}\left(U_{\alpha}\right) ; L\right)=0$ for any open set $U_{\alpha}$ of $x_{\alpha}$. We note that $\pi_{\alpha}^{-1}\left(U_{\alpha}\right) \simeq \underset{\alpha<\beta}{\lim } \pi_{\alpha, \beta}^{-1}\left(U_{\alpha}\right)$, and by continuity property of sheaf cohomology $([1], p .72)$,

$$
h_{c}^{n+1}\left(\pi_{\alpha}^{-1}\left(U_{\alpha}\right) ; L\right) \approx \lim _{\rightarrow} H_{c}^{n+1}\left(\pi_{\alpha, \beta}^{-1}\left(U_{\alpha}\right) ; L\right)=0,
$$

because for each $\alpha, H_{c}^{n+1}\left(U_{\alpha} ; L\right)=0$ for any open subset $U_{\alpha}$ of $X$. Now we prove that $H_{c}^{n+1}(U ; L)=0$ for any open subset $U$ of $X$. If not, suppose

$$
0 \neq s \in H_{c}^{n+1}(U ; L) \approx \lim _{\rightarrow} H_{c \mid K}^{n+1}(U ; L)
$$

where $K$ runs over all compact subsets of $U$. For some compact subset $K$ contained in $U$, let $0 \neq s_{k} \in H_{c \mid K}^{n+1}(U ; L)$ represent $s$. Since $c \mid K$ is the family cld of all closed subsets of $K$ and $K$ is $c$-taut in $U$, we have that

$$
H_{c \mid K}^{*}(U, K ; L) \approx H_{(c \mid K) \mid U K}^{*}(U ; L)=0
$$

Then from the exact cohomology sequence for the pair $(U, K)$ with supports $c$, it follows that

$$
H_{c \mid K}^{*}(U ; L) \approx H_{c l d}^{*}(K ; L)
$$

Thus $s_{k} \in H_{c l d}^{n+1}(K ; L)$ represents $s \in H_{c}^{n+1}(U ; L)$. Using the directedness property of the indexing set $\Omega$, it is easy to verify that for any compact subset $K$ of $X$ we can find an open set $U_{\beta}$ of $X_{\beta}$ for some $\beta \in \Omega$ such that $K \subseteq \pi_{\beta}^{-1}\left(U_{\beta}\right) \subseteq U$. Therefore the restriction homomorphism

$$
H_{c}^{n+1}(U ; L) \rightarrow H_{c l d}^{n+1}(K ; L)
$$

factors through $H_{c}^{n+1}\left(\pi_{\beta}^{-1}\left(U_{\beta}\right) ; L\right)=0$. This implies that $s_{k}=0$, a 
contradiction.

3. Failure of expansion theorem for $\operatorname{dim}_{L}$

Next we consider the following question: what are those spaces $X$ for which $\operatorname{dim}_{L}(X)$ is independent of $L ?$ the following theorem gives an answer.

THEOREM 2. (a) If $X$ is a CW-complex, then $\operatorname{dim}_{L}(X)$ is independent of $L$; in fact the combinatorial dimension of $X=\operatorname{dim} X=\operatorname{dim}_{L}(X)$ for each $L$.

(b) If $X$ is locally compact and paracompact such that $\operatorname{dim} X \leq 1$, then $\operatorname{dim} X=\operatorname{dim}_{L}(X)$ for each $L$.

(c) There exists a compact metric space $X$, such that $\operatorname{dim} X=2$, and (b) above fails.

Proof. (a) By the disjoint sum theorem [5] and induction on the skeletons of $X$, it follows that for any ring $L, \operatorname{dim}_{L}(X)$ is less than of equal to the combinatorial dimension of $X=\operatorname{dim} X$. Conversely, suppose $\sigma$ is an arbitrary $n$-cell of $x$. Then $\sigma$ lo contains an open set of $X$ homeomorphic to the euclidean space $\mathbf{R}^{n}$. For any ring $L$, however, $\operatorname{dim}_{L}\left(\mathbb{R}^{n}\right)=n \quad([1]$, p. 144). Hence by the subset theorem of the cohomological dimension $\operatorname{dim}_{L}(X) \geq n$. This shows that for any ring $L$, $\operatorname{dim}_{L}(X)$ is greater than or equal to the combinatorial dimension of $X$, completing the proof.

(b) The case when $\operatorname{dim} X=0$ is obvious. Hence we assume that $\operatorname{dim} X=1$. If $X$ is locally compact and paracompact, then there exists a locally finite cover $\left\{k_{\alpha}\right\}$ consisting of compact subsets of $X$. Hence $X$ has the Morita weak topology with respect to the family $\left\{K_{\alpha}\right\}$. Therefore, by the weak topology sum theorem for $\mathrm{dim}_{L}$ [5], $\operatorname{dim}_{L}(X)=\sup _{\alpha}\left\{\operatorname{dim}_{L}\left(K_{\alpha}\right)\right\}$ for any $L$. A similar theorem for covering dimension also holds. We have only to prove that $\operatorname{dim}_{L}(X) \geq \operatorname{dim} X$ for 
each $L$. Suppose for some $L, \operatorname{dim}_{L}(X)<\operatorname{dim} X=1$. Then $\operatorname{dim}_{L}\left(K_{\alpha}\right)=0$ for each $\alpha$. This implies that $K_{\alpha}$ must be totally disconnected ([5], Proposition 3.6). But then compactness of $K_{\alpha}$ means $\operatorname{dim} K_{\alpha}=0$ for each $\alpha$. Hence by the sum theorem mentioned above $\operatorname{dim} X=0$, a contradiction.

(c) There are many such examples. However, we describe here a simple one. Let $a$ be a positive integer and $D$ be the 2-disc. On the boundary $S^{l}$ of $D$ identify points which correspond under the rotation through an angle $2 \pi / \alpha$. Let $M(a)$ denote the quotient space and $h: D \rightarrow M(a)$ be the quotient map. Let $T$ denote the set $h\left(S^{1}\right)$. Then clearly $M(a)$ is a simplicial 2-polytope. We give it a fixed triangulation. For any 2-simplex $s$ of $M(a)$ if $\dot{s}$ denotes the boundary of $s$, then there is an obvious map $f:(M(a), T) \rightarrow(s, \dot{s})$ such that $f \mid T$ is a homeomorphism and $f(M(a) \backslash T) \subseteq s \backslash \dot{s}$. We easily see that $H^{2}(M(\alpha), T) \approx \mathbb{Z}$ and if $\alpha$ is a generator of this group, then $f^{*}$ maps a generator of $H^{2}(s, \dot{s})$ to $\pm a^{\alpha}$. Also if $a>1$, then the coboundary homomorphism $H^{\mathcal{l}}(T) \rightarrow H^{2}(M(a), T)$ is non-trivial. Now let $\underline{\underline{\mathrm{A}}}=\left\{a_{1}, a_{2}, \ldots, a_{n}, \ldots\right\}$ be a sequence of positive integers with $a_{i}>1$ for each $i$. Put $M\left(a_{1}\right)=M_{1}$ and suppose for each $i \leq k$, a simplicial 2-polytope $M_{i}$ and mappings $\pi_{i-1, i}: M_{i}+M_{i-1}$ have been constructed. Let $\sigma_{h}, h=1,2, \ldots, n_{k}$, be all 2-simplices of $M_{k}$. Replace each $\sigma_{h}$ by $M_{h}\left(a_{k+1}\right)$ such that

$$
M_{h}\left(a_{k+1}\right) \cap M_{h^{\prime}}\left(a_{k+1}\right)=T_{h} \cap T_{h^{\prime}}=\sigma_{h} \cap \sigma_{h^{\prime}}, h \neq h^{\prime} .
$$

Here $T_{h}$ and $T_{h}$, are the boundaries of $M_{h}\left(a_{k+1}\right)$ and $M_{h},\left(a_{k+1}\right)$ respectively. Define $M_{k+1}=\bigcup_{h=1}^{n_{k}} M_{h}\left(a_{k+1}\right)$. Then $M_{k+1}$ is a simplicial 2-polytope and there is a mapping $\pi_{k, k+1}: M_{k+1} \rightarrow M_{k}$ such that if $\Delta_{k}$ is the 1-skeleton of $M_{k}$, then $\pi_{k, k+1} \mid\left(\pi_{k, k+1}\right)^{-1}\left(\Delta_{k}\right)$ is a homeomorphism. Now we have an inverse sequence $\left\{M_{k}, \pi_{k-1, k}\right\}$ of 2-polytopes. Let $M(\underline{\underline{A}})$ 
denote the inverse limit of this sequence and $\pi_{k}: M(\underline{\underline{A}}) \rightarrow M_{k}$ be the canonical maps. Clearly $\pi_{k} \mid \pi_{k}^{-1}\left(\Delta_{k}\right)$ is a homeomorphism. We call $T=\pi_{1}^{-1}\left(T_{1}\right)$ the boundary of $M(\underline{\underline{A}})$ where $T_{1}$ is the boundary of $M\left(a_{1}\right)$.

Since the inverse limit of an inverse sequence of metrizable spaces is metrizable, $M(\underline{\underline{A}})$ is a compact metrizable space. By Theorem $I$, $\operatorname{dim}_{\mathbb{Z}}(M(\underline{\underline{A}})) \leq 2$. Also $H^{l}\left(T_{i}\right) \rightarrow H^{2}\left(M\left(a_{i}\right), T_{i}\right)$ is non-trivial for each $i$. Let $\alpha$ be a generator of $H^{l}\left(M(\underline{\underline{A}}), T_{1}\right)$, put

$$
\pi_{1, k}=\pi_{1,2} \circ \ldots \circ \pi_{k-1, k} \text { and } T_{k}=\left(\pi_{1, k}\right)^{-1}\left(T_{1}\right) \text {. }
$$

Take an oriented 2-simplex $\tau$ of $M_{k}$ and denote by $\alpha_{\tau}$ the element of $H^{2}\left(M_{k}, T_{k}\right)$ which is represented by the cocycle taking 1 on $\tau$ and 0 on other 2-simplices. Then $\alpha_{\tau}$ is of infinite order and $\left(\pi_{1, k}\right)^{*}(\alpha)= \pm\left(a_{2} a_{3} \ldots a_{k}\right) \alpha_{\tau}$. Thus the generator of $H^{1}(T)$ will go to an element of infinite order under the induced coboundary homomorphism $H^{l}(T) \rightarrow H^{2}(M(\underline{A}), T)$, that is, this map is non-trivial. Consequently, the map $\pi \mid T: T \rightarrow T_{1} \simeq S^{l}$ cannot be extended to $M(\underline{\underline{A}})$. This shows that $\operatorname{dim}_{\mathbb{Z}}(M(\underline{\underline{A}}))>1$, and so $\operatorname{dim}_{\mathbb{Z}}(M(\underline{\underline{A}}))=2$ for any such sequence $\underline{\underline{\mathrm{A}}}=\left\{a_{1}, a_{2}, \ldots, a_{n}, \ldots\right\}$.

Next, let us choose

$$
\underline{\underline{A}}=\left\{q^{k} \mid q \text { runs over all positive primes and } k \in \mathbf{N}\right\} \text {. }
$$

In this case we claim that for any given prime $p, \operatorname{dim}_{\mathbb{Z}_{p}}(M(\underline{\underline{A}}))=1$. It suffices to show that for any 2-simplex $\sigma$ of $M_{k}$, the homomorphism

$$
\left(\pi_{k} \mid \pi_{k}^{-1}(\sigma)\right)^{*}: H^{2}\left(\sigma, \dot{\sigma} ; \mathbb{Z}_{p}\right) \rightarrow H^{2}\left(\pi_{k}^{-1}(\sigma), \pi_{k}^{-1}(\dot{\sigma}) ; \mathbb{Z}_{p}\right)
$$

is trivial. But this follows at once because we can choose 1 larger than $k$ so that $a_{1}$ is a power of $p$ and 


$$
\left(\pi_{k, 1}\right)^{*}: H^{2}\left(\sigma, \dot{\sigma} ; \mathbb{Z}_{p}\right)+H^{2}\left(\left(\pi_{k, 1}\right)^{-1}(\sigma),\left(\pi_{k, 1}\right)^{-1}(\dot{\sigma}) ; \mathbb{Z}_{p}\right)
$$

is the multiplication by $\pm a_{k} a_{k+1} \cdots a_{1}$ on generators. Hence the generator of $H^{2}\left(\sigma, \dot{\sigma} ; \mathbb{Z}_{p}\right)$ must be mapped to zero element of the limit group $H^{2}\left(\pi_{k}^{-1}(\sigma), \pi_{k}^{-1}(\dot{\sigma}) ; \mathbb{Z}_{p}\right)$.

Finally, we have our main result.

THEOREM 3. Suppose $X$ is any compact metris space for which there exists a ring $L$ (not necessarily a PID) such that $\operatorname{dim}_{L}(X)<\operatorname{dim}_{\mathbb{Z}}(X)$ (for example, $M(\underline{\underline{A}})$ of Theorem 2 (c)). Then $X$ cannot be expressed as the inverse limit of an inverse system $\left\{K_{\alpha}, \pi_{\alpha, \beta}\right\}$ of compact polyhedra $K_{\alpha}$ such that $\operatorname{dim}_{L}\left(K_{\alpha}\right) \leq \operatorname{dim}_{L}(X)$.

Proof. Suppose $\operatorname{dim}_{\mathbb{Z}}(X)=n$ and $\operatorname{dim}_{L}(X)=k<n$. Let $\left\{k_{\alpha}, \pi_{\alpha, \beta}\right\}$ be an inverse system of compact polyhedra $K_{\alpha}$ such that $\operatorname{dim}_{L}\left(K_{\alpha}\right) \leq k$ for each $\alpha$, and $X$ is homeomorphic to ${ }^{\lim } K_{\alpha}$. Then by Theorem $2(a)$, $\operatorname{dim} \mathbb{Z}_{\alpha}\left(K_{\alpha}\right) \leq k$ for each $\alpha$. Hence, by Theorem 1 , $\operatorname{dim}(X) \leq k$, a contradiction.

\section{References}

[1] Glen E. Bredon, Sheaf theory (McGraw-Hill, New York, Toronto, London, 1968).

[2] M.G. Charalambous, "The dimension of inverse limit and $N$-compact spaces", Proc. Amer. Math. Soc. 85 (1982), 648-652.

[3] Satya Deo, "A one dimensional manifold is of cohomological dimension 2 ", Proc. Amer. Math. Soc. 52 (1975), 445-446.

[4] Satya Deo, "The cohomological dimension of a $n$-manifold is $n+1$ ", Pacific J. Math. 67 (1976), 155-160.

[5] Satya Deo and R.A. Shukla, "On sum theorems of cohomological dimension", Acta Math. Acad. Sci. Hungar. (to appear). 
[6] Yûkiti Katuta, "On the covering dimension of inverse limits", Proc. Amer. Math. Soc. 84 (1982), 588-592.

[1] Sibe Mardešić, "On covering dimension and inverse limits of compact spaces", Illinois J. Math. 4 (1960), 278-291.

[8] Keiô Nagami, "Dimension of non-normal spaces", Frond. Math. 109 (1980), $113-121$.

[9] A.R. Pears, Dimension theory of general spaces (Cambridge University Press, England, New York, Melbourne, 1975).

Department of Mathematics,

University of Jammu,

Canal Road,

Jammu 180001,

India. 